\title{
EDITORIAL
}

\section{Contemporary considerations for risk stratification, sudden death and prevention in hypertrophic cardiomyopathy}

\author{
B J Maron
}

Heart 2003;89:977-978

For patients with hypertrophic cardiomyopathy, the likelihood of receiving primary prevention for sudden death with an ICD may depend in part on the country of residence

Correspondence to: Barry J M aron, M D M inneapolis Heart Institute Foundation, 920 E 28 th Street, Suite 60, M inneapolis, M N 55407, USA; hcm.maron@mhif.org
S udden death has been the most devastating, un predictable and recognisable complication of hypertrophic cardiomyopathy (HCM), since the modern description of the disease by Teare over 40 years ago. ${ }^{1} \mathrm{HCM}$ is now recognised as the most common cause of sudden cardiac death in young people, including trained athletes, ${ }^{2}{ }^{3}$ although only a minority of all HCM patients are susceptible to the risk of sudden death (perhaps 10-15\%). ${ }^{4}$ Nevertheless, the identification of this patient subset has been a priority, and the subject of a considerable body of literature. ${ }^{3-10}$ Because of the power and availability of the implantable cardioverter-defibrillator (ICD) for both primary and secondary prevention of sudden death, and its recent application to $\mathrm{HCM},{ }^{9}$ it now more important than ever to identify those patients within this broad disease spectrum who are at high risk for a catastrophic event.

RISK STRATIFICATION PROFILES

Defining reliable clinical markers for sudden death, and isolating the minority of HCM patients who are at high risk, has been an imposing task. This has been due largely to the relatively low prevalence of $\mathrm{HCM}$ within cardiology practice and its striking heterogeneity in clinical expression and outcome. ${ }^{310}$ Indeed, there is not yet a single test which is generally accepted as capable of assessing the risk level in all HCM patients with precision.

Because HCM patients who are at risk for sudden death are often asymptomatic, risk factors assessed non-invasively have been most practical in clinical terms. The highest risk profiles in HCM are presently identified by one or more clinical markers $^{3-6}$ : (1) prior cardiac arrest; (2) family history of HCM related death; (3) otherwise unexplained syncope; (4) frequent or prolonged bursts of non-sustained ventricular tachycardia on Holter; (5) abnormal blood pressure response to exercise; and (6) extreme left ventricular hypertrophy.

It has been proposed, based on genotypephenotype correlations, that disease causing mutations in genes encoding sarcomeric proteins could also be unfavourable (or favourable) primary determinants of sudden death. ${ }^{7}$ While such a molecular based treatment strategy is attractive, ${ }^{7}$ caution is warranted in translating the "good gene/bad gene" hypothesis to the practical clinical management of HCM patients. ${ }^{8}$ Available epidemiologic-genetic data are based on relatively small numbers of genotyped families; each of the 10 known disease causing HCM mutant genes (and approximately 200 individual mutations) occur infrequently in the general population of this genetically heterogeneous disease. Furthermore, genotyping involves complex, time consuming, and expensive laboratory techniques which are presently confined to only a select few research oriented laboratories. ${ }^{3}$

Other non-invasive indices such as signal averaged ECG and QT dispersion have not proven to be useful for the stratification of risk. ${ }^{11}$ Therefore, there is a continuing aspiration to develop additional tests to better define the high risk HCM patient. The recently reported study by Butera and colleagues, ${ }^{12}$ comprising a relatively small series of children with HCM who demonstrated a high rate of sudden death, is timely by raising the possibility that substantial heart rate variability (reflecting autonomic imbalance) may be predictive of high risk in this disease. Whether heart rate variability will ultimately achieve inclusion into the risk stratification armamentarium of HCM will require confirmatory studies in larger patient populations.

\section{PREVENTION OF SUDDEN DEATH}

Historically, treatment strategies to reduce the likelihood of sudden death in HCM have been largely pharmacologic, predicated on prophylactic administration of $\beta$ blockers, verapamil, type IA antiarrhythmic agents (that is, quinidine, procainamide), and amiodarone to patients judged to be at unacceptably high risk. ${ }^{13}$ However, these drugs have never been shown to mitigate sudden death risk convincingly, usually cannot be tolerated over the long periods of risk incurred by young HCM patients because of their potential toxicity, and therefore seem outdated compared to the power of the ICDs. ${ }^{9}$

When sudden death risk is judged by available clinical evidence to be unacceptably high in HCM patients, the ICD has proven to be the most effective and reliable treatment option, harbouring the potential to alter the natural history of this disease. $^{9}$ In a multicentre retrospective study, which has been a stimulus to many device implants in HCM since its publication in February 2000, ICDs aborted potentially lethal ventricular tachyarrhythmias in almost $25 \%$ of the patients 


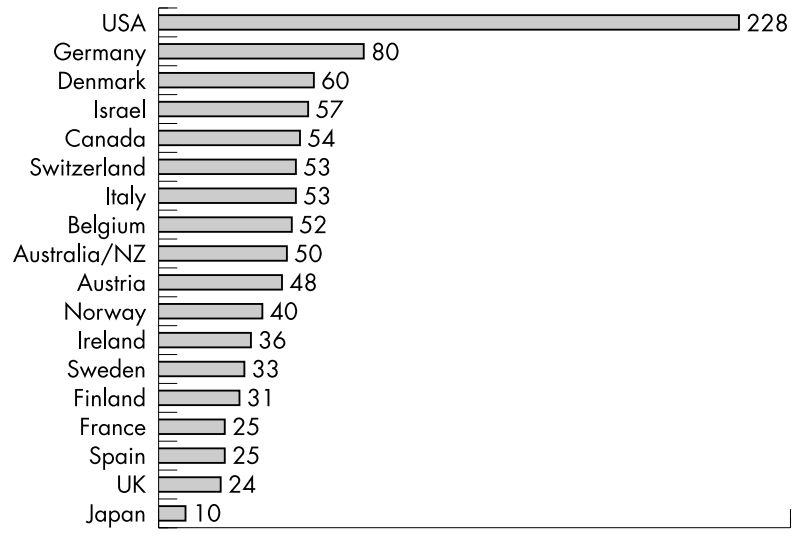

ICDs per million population

Figure 1 Defibrillator implants worldwide in the year 2001, shown by country (per one million population). The rates shown are for all ICD indications, but largely represent implants in patients with coronary artery disease.

over only a three year observation period. ${ }^{9}$ Appropriate ICD interventions occurred at $11 \%$ per year for secondary prevention (implant following cardiac arrest) and at about 5\% per year for primary prevention (implant for non-invasive risk factors), usually in young patients with no or only mild symptoms. Of note, ICDs often remained dormant for prolonged periods of time (up to nine years) before discharging, ${ }^{9}$ emphasising the unpredictable timing of sudden death events and the particularly long risk period characteristic of high risk HCM patients. ${ }^{14}$

The ICD is strongly warranted in HCM for secondary prevention. ${ }^{15}$ However, implants for primary prevention of sudden death represent a novel treatment strategy for which there is not yet complete acceptance. This is due largely to the uncertainty as to precisely which HCM patients are at greatest risk and most deserving of such intervention. ${ }^{3}$ While multiple clinical risk factors convey increasing risk for sudden death, ${ }^{4}$ and patients without risk factors rarely die suddenly, ${ }^{4}$ it is also clear that in selected patients a single major risk factor may well be of sufficient magnitude to justify aggressive treatment with the ICD for primary prevention of sudden death. ${ }^{359} \mathrm{At}$ present, while some investigators (particularly in the USA) ${ }^{3}$ favour strong consideration for an ICD in the presence of one major risk factor, others (largely European investigators) have taken a more conservative and restrictive stance requiring at least two risk factors before recommending a prophylactic ICD. ${ }^{46}$ However, all agree that such management decisions best serve patients by integrating individual clinical judgement with the overall risk profile, including age, strength of the risk factor(s) identified, and most importantly the desires of the patient and family after being fully informed regarding the advantages (and potential complications) of defibrillator therapy.

Indeed, it may be very difficult in a heterogeneous and relatively uncommon disease such as $\mathrm{HCM}^{3810}$ to establish practice guidelines for ICD related decisions based solely on the construction of strict algorithms and arbitrary cutpoints. ${ }^{4}$ Such a strategy could result in the under treatment of some HCM patients truly at risk for sudden death. For exam- ple, in formulating a risk level for surviving relatives in a family affected by HCM , how many sudden deaths are sufficient to constitute a risk factor? One or two? If two deaths are required, as proposed by some investigators, ${ }^{46}{ }^{15}$ should one relative be required to wait until another relative dies before an ICD is considered? In an HCM family with two young affected brothers (one who has already died suddenly of $\mathrm{HCM}$ ), would it not be appropriate to offer the option of a prophylactic ICD to the surviving brother, even in the absence of any other independent risk factors? These are just a few of the innumerable and difficult (if not impossible) questions which commonly arise in contemplating primary prevention of sudden death in individual HCM patients.

It is also of note that physician and patient attitudes toward ICDs (and access to such devices within the respective health care system) can vary considerably among countries and cultures (fig 1), ${ }^{16}$ thereby impacting importantly on clinical decision making and specifically the threshold for prophylactic implants in HCM patients. For example, employment of ICDs relative to population size is much higher in the USA, accounting for about $75 \%$ of all implants worldwide-fivefold greater than in Europe and 10 times that of the UK (fig 1). ${ }^{16}$ Such disparity in implant rates suggests that for HCM patients with equivalent levels of risk, the likelihood of receiving primary prevention for sudden death with an ICD would be highly dependent on the patient's country of residence.

\section{REFERENCES}

1 Teare D. A symmetrical hypertrophy of the heart in young adults. Br Heart J 1958;20:1-18.

2 Maron BJ, Shirani J, Poliac LC, et al. Sudden death in young competitive athletes: clinical, demographic and pathological profiles. JAMA 1996;276:199-204.

3 Maron BJ. Hypertrophic cardiomyopathy: a systematic review. JAMA 2002:287:1308-20.

4 Elliott PM, Poloniecki J, Dickie S, et al. Sudden death in hypertrophic cardiomyopathy: identification of high risk patients. J Am Coll Cardiol 2000;36:2212-18

5 Spirito $P$, Bellone $P$, Harris KM, et al. M agnitude of left ventricular hypertrophy predicts the risk of sudden death in hypertrophic cardiomyopathy. N Engl] Med 2000;342:1778-85.

6 Elliott PM, Gimeno JR, M ahon N G, et al. Relation between severity of left-ventricular hypertrophy and prognosis in patients with hypertrophic cardiomyopathy. Lancet 2001;357:420-4.

7 Watkins $\mathrm{H}$. Sudden death in hypertrophic cardiomyopathy [editorial]. $N$ EnglJ Med 2000;372:422-3.

8 Ackerman MJ, Van Driest SL, 0 mmen SR, et al. Prevalence and age-dependence of malignant mutations in the beta-myosin heavy chain and troponin $\mathrm{T}$ genes in hypertrophic cardiomyopathy. A comprehensive outpatient perspective. I Am Coll Cardiol 2002;39:2042-8.

9 Maron BJ, Shen W -K, Link M S, et al. Efficacy of implantable cardioverter-defibrillators for the prevention of sudden death in patients with hypertrophic cardiomyopathy. N EnglJ Med 2000;342:365-73.

10 Wigle ED, Rakowski H, Kimball BP, et al. Hypertrophic cardiomyopathy: clinical spectrum and treatment. Circulation 1995;92:1680-92.

11 Maron BJ, Leyhe MJ III, Casey SA, et al. Assessment of Q T dispersion as a prognostic marker for sudden death in a regional non-referred hypertrophic cardiomyopathy cohort. Am J Cardiol 2001;87:114-15.

12 Butera G, Bonnet D, Kachaner J, et al. Heart rate variability in children with hypertrophic cardiomyopathy. Heart 2003:89:205-6.

13 McKenna WJ, 0 akley CM, Krikler DM, et al. Improved survival with amiodarone in patients with hypertrophic cardiomyopathy and ventricular tachycardia. Br Heart J 1985;53:412-16

14 Maron BJ, O livotto I, Spirito P, et al. Epidemiology of hypertrophic cardiomyopathy-related death. Revisited in a large non-referral-based patient population. Circulation 2000;102:858-64

15 Priori SG, Aliot E, Blomstrom-Lundqvist C, et al. Task force on sudden cardiac death of the European Society of Cardiology. Eur Heart J $2001 ; 22: 1374-450$

16 Camm AJ, N isam S. The utilization of the implantable defibrillator: a European enigma. Eur HeartJ 2000;21:1998-204. 